Physics

Physics Research Publications

\title{
Quantum interference effects in particle transport through square lattices
}

E. Cuansing H. Nakanishi 


\title{
Quantum interference effects in particle transport through square lattices
}

\author{
E. Cuansing* and H. Nakanishi \\ Department of Physics, Purdue University, West Lafayette, Indiana 47907, USA
}

(Received 26 June 2004; published 28 December 2004)

\begin{abstract}
We study the transport of a quantum particle through square lattices of various sizes by employing the tight-binding Hamiltonian from quantum percolation. Input and output semi-infinite chains are attached to the lattice either by diagonal point-to-point contacts or by a busbar connection. We find resonant transmission and reflection occurring whenever the incident particle's energy is near an eigenvalue of the lattice alone (i.e., the lattice without the chains attached). We also find the transmission to be strongly dependent on the way the chains are attached to the lattice.
\end{abstract}

DOI: 10.1103/PhysRevE.70.066142

PACS number(s): 05.60.Gg, 05.50.+q, 05.10.-a, 73.23.-b

Quantum interference effects are important in the transport of particles in mesoscopic systems. Consider, for example, a particle traversing a square array of quantum dots. Assume the distance between dots is close enough so that the particle can hop between nearest-neighbor dots. Considering only the effect of quantum interference, will the particle go through the lattice? Classically, the particle has a multitude of paths to go from one end of the lattice to the other, depending on the size of the lattice. Quantum mechanically, however, constructive or destructive interference can occur because of the different path lengths. Thus, the transmission of a particle is not assured even when there are classically well-defined paths for it to go through the lattice. In this work we investigate the effects of quantum interference in the transport of a particle in discrete and finite square lattices.

We consider the particle to be governed by the tightbinding Hamiltonian from quantum percolation $[1,2]$. This Hamiltonian has the form

$$
H=\sum_{\langle i j\rangle} v_{i j}(|i\rangle\langle j|+| j\rangle\langle i|),
$$

where $|i\rangle$ and $|j\rangle$ represent tight-binding basis functions centered on sites $i$ and $j$, respectively, and $v_{i j}=1$ if $i$ and $j$ are nearest neighbors and $v_{i j}=0$ otherwise. The sum is only over nearest neighbors. In quantum percolation the particle is confined to traverse through disordered clusters constructed from the methods of percolation theory [3] with some occupation probability $p$. For $p<1$ there is disagreement whether particle states are localized or extended. In a review by Mookerjee et al. [4], they concluded that all states are localized and transport is dominated by statistically exceptional necklacelike resonant states. Daboul et al. [5], by calculating the moments of distances between pairs of lattice sites using series expansion methods, found evidence of a transition from exponentially localized to extended or power-law decaying states with an energy-dependent occupation probability threshold $p_{q}(E)$. Recent numerical studies of the scaling of the conductance $g$ by Hałdaś et al. [6], however, found all

\footnotetext{
*Department of Physics and Astronomy, University of Pittsburgh, Pittsburgh, PA 15260.
}

states to be localized and no indication of a localizationdelocalization transition. To properly interpret the results of any calculation on the question of the existence of such a transition as a function of the degree of disorder $p$, however, we need to know the behavior of the transmittance in the limit $p \rightarrow 1$. In this work we thus consider the limiting case $p=1$ wherein all sites in the lattice are occupied. We are going to show that even in this limit, the transport through the lattice is very sensitive to the incident particle's energy, varying from complete transmission to complete reflection, and also to the type of coupling between the lattice and the input and output chains.

To determine the transport properties of a particle traversing the square lattice, we attach semi-infinite chains to the left and right sides of that lattice. Call the left semi-infinite chain the input chain and the right semi-infinite chain the output chain. The particle is made incident to the lattice via the input chain. If the particle goes through the lattice, then it exits via the output chain. Following the Landauer-Büttiker formalism [7], the conductance of the system can then be determined from the resulting transmission and reflection amplitudes. Because of the semi-infinite chains, the corresponding matrix equation resulting from Eq. (1) is also infinite. Daboul et al. [5] recently described a method to transform the infinitely sized Hamiltonian matrix in Eq. (1) into a reduced matrix $H^{\prime}$ that is finite and involves only the lattice and its connections to the semi-infinite chains using an ansatz. We are implementing this method in this work.

There are various ways of attaching the semi-infinite chains to the square lattice. In this work we consider two complimentary ways. One is by point-to-point contacts and the other is by a busbar connection. In point-to-point contacts the input chain is singly attached to the top-leftmost site while the output chain is singly attached to the bottomrightmost site of the square lattice. In a busbar connection the input chain is attached to all the sites in the left edge of the lattice while the output chain is attached to all the sites in the right edge of the lattice. This connection roughly corresponds to a physical configuration where the contacts are good conductors and are large enough to span a macroscopic fraction of the sites along the edges of the lattice. The two types of couplings we have chosen are complimentary. This is because the single connections in point-to-point diagonal contacts preserve the bipartite symmetry of the square lattice while the multiple connections in the busbar connection destroy that symmetry. 


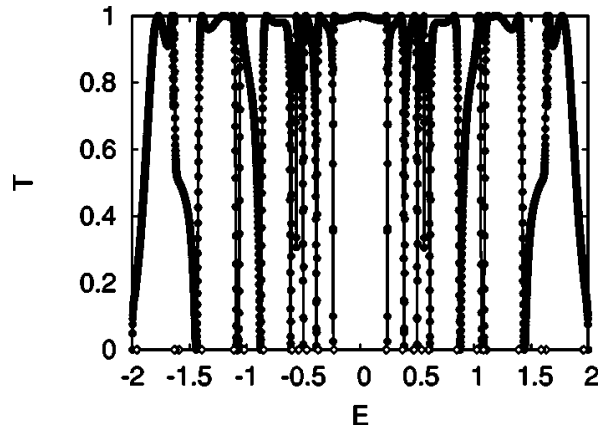

FIG. 1. Plot of the transmission coefficient $T$ against the incident particle's energy $E$ for a $10 \times 10$ lattice with point-to-point contacts to the input and output chains. The diamonds $(\diamond)$ are the locations of the doubly degenerate eigenvalues of the isolated square lattice.

Daboul et al. [5] proposed the following ansatz:

$$
\begin{gathered}
\psi_{-(n+1)}=e^{-i n q}+r e^{i n q}, \\
\psi_{+(n+1)}=t e^{i n q},
\end{gathered}
$$

where $n=0,1,2, \ldots$. The $\psi_{-(n+1)}$ represent components of the wave function along the input chain and the $\psi_{+(n+1)}$ represent components along the output chain. $\psi_{-1}$ and $\psi_{+1}$ are for sites in the input and output chains, respectively, that are directly connected to the lattice. The ansatz restricts solutions to Eq. (1) in the form of incident and reflected plane waves along the input chain and transmitted plane waves along the output chain. Because of this ansatz, the energy of the incident particle is also restricted to be within $E=-2$ and $E=2$. The transmission and reflection coefficients can be determined from the $t$ and $r$ in Eq. (2) by $T=|t|^{2}$ and $R=|r|^{2}$.

Once the Hamiltonian matrix in Eq. (1) is reduced to $H^{\prime}$, the resulting problem can then be cast into the form of a linear equation $\left(H^{\prime}-E\right) \psi=\gamma$, where $\gamma$ is solely a function of $E$. This linear equation can then be solved for $\psi$ once $E$ is chosen. We determine $t$ and $r$ from $\psi$ by numerically solving the above linear equation exactly-i.e., from $\psi=\left(H^{\prime}-E\right)^{-1} \gamma$. The matrix $\left(H^{\prime}-E\right)$ is sparse and is numerically very close to being singular, making the use of standard methods such as the LU decomposition, wherein the matrix is decomposed into a lower triangular matrix $L$ and an upper triangular matrix $U$, fail in some instances. As such, we implement the technique called singular value decomposition [8] to carefully determine the inverse of $\left(H^{\prime}-E\right)$.

Shown in Fig. 1 is a plot of the transmission coefficient against the incident particle's energy for a $10 \times 10$ lattice with point-to-point contacts to the input and output chains. Also shown are the locations of the doubly degenerate eigenvalues of the isolated square lattice. An isolated lattice is one where the input and output chains are not attached. The system is highly transmitting except at some values of energy where there are sharp dips and the system becomes highly reflecting. Notice that the dips occur near the eigenvalues of the isolated lattice. This phenomenon is analogous to resonant tunneling [9] of an incident particle through, for ex-
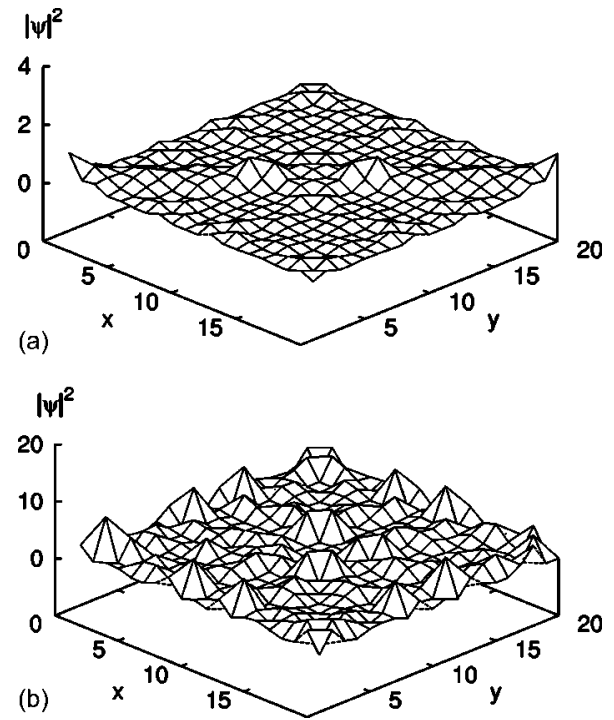

FIG. 2. Sample states of a particle traversing a $20 \times 20$ lattice with point-to-point contacts to the input and output chains. (a) Highly transmitting state with $E=0.39$. (b) Highly reflecting state with $E=0.41$.

ample, a double-barrier system. In such a system a peak in particle transmission occurs whenever the incident particle's energy is the same as the energy of one of the well's bound states. In this work, however, although there is no tunneling involved, we do see resonant reflection whenever the energy of the incident particle falls near an eigenvalue of the isolated lattice.

Notice as well that there is symmetry between the $E>0$ side and the $E<0$ side. The square lattice has bipartite symmetry and point-to-point contact connections preserve that symmetry. Maintaining bipartite symmetry can in turn be shown to ensure the symmetry in $T$ about $E=0$.

As the size of the isolated lattice is increased the number of its associated eigenvalues will also increase. For the lattice with point-to-point contacts to the chains, we also see more dips in the transmission coefficient as we increase the size of the lattice. These dips are also located near the doubly degenerate eigenvalues of the corresponding isolated square lattice.

Shown in Figs. 2(a) and 2(b) are sample states that are highly transmitting and highly reflecting, respectively, for a particle traversing a $20 \times 20$ lattice. The lattice is at the $x y$ plane. The input chain is attached to the site in the lattice located at $(1,1)$. The output chain is attached to the site in the lattice located at $(20,20)$. The $z$ axis is the absolute square of the components of the wave function at each corresponding lattice site, $|\psi(x, y)|^{2}$. For the highly transmitting state we see a diagonal line of nonzero $\psi$ going from the input to the output chains. Though this is not always true for all highly transmitting states, those with this feature are always highly transmitting. In the highly reflecting state, on the other hand, we see large fluctuations and destructive interference is manifest at the input and output sites.

Shown in Fig. 3 is the transmission $T$ versus the incident particle's energy $E$ plot for a $10 \times 10$ lattice with busbar connections to the input and output chains. In contrast to the 


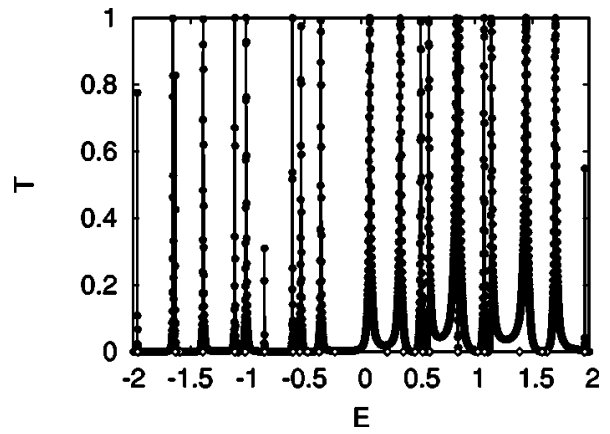

FIG. 3. Plot of $T$ against $E$ for a $10 \times 10$ lattice with busbar connections to the input and output chains. The diamonds $(\diamond)$ are again the locations of the doubly degenerate eigenvalues of the isolated square lattice.

case for point-to-point contacts, the system is mostly reflecting but with sharp peaks in transmission at certain values of the incident particle's energy. Also shown in Fig. 3 are the locations, as diamonds, of the doubly degenerate eigenvalues of the corresponding isolated $10 \times 10$ square lattice. Notice that for the $E<0$ side the locations of the transmission peaks are near the eigenvalues of the isolated lattice. This is similar to the case with point-to-point contacts but instead of resonant reflection we see resonant transmission. For the $E>0$ side, however, some of the peaks do not coincide with the locations of the eigenvalues of the isolated lattice.

From the ansatz shown in Eq. (2), the wave vector $q$ of the particle is related to its energy by $E=2 \cos (q)$, where $q$ $=2 \pi / \lambda$. For negative energies, the particle's wavelength is constrained to be within $\frac{4}{3}<\lambda<4$. For positive energies, the wavelength should be within either $\lambda<\frac{4}{3}$ or $\lambda>4$. Unlike the case for point-to-point contacts, the lack of symmetry between the $E<0$ and $E>0$ sides of the plot in Fig. 3 indicates the significance of the incident particle's wavelength when undergoing through a busbar connection. Mathematically, the multiple connections of the busbar destroys the bipartite symmetry of the square lattice and, consequently, destroying the symmetry in $T$ about $E=0$.

Let us call those sites at the sides of the lattice that are directly connected to the input and output chains as belonging to the input and output connection boundaries, respectively. Because of the multiple connections in a busbar, destructive interference can occur at the connection boundaries, resulting in a vanishingly small transmission through the lattice. Some of the minima in transmission in Fig. 3 appear to be consistent with rules analogous to optical-interference minima-maxima conditions on the boundary. For example, the condition that an integer number of wavelengths fit within the boundary of a lattice of size $L \times L$-i.e., the condition $L-1=n \lambda$-would suggest that certain values of $\lambda$ result in destructive interference. This would include $\lambda=1$-i.e., $E=2$ from $E=2 \cos (2 \pi / \lambda)$-for all $L$ and $\lambda=2$ $(E=-2)$ for all odd values of $L$. In actuality, completely destructive interference occurs when $E=2$ for all $L>2$ and also when $E=-2$ for all $L \neq 2$ and 4 . There are also several other minima in $T$ that are consistent with this condition. For example, for $L=5, \lambda=4(E=0)$ also satisfies the condition and
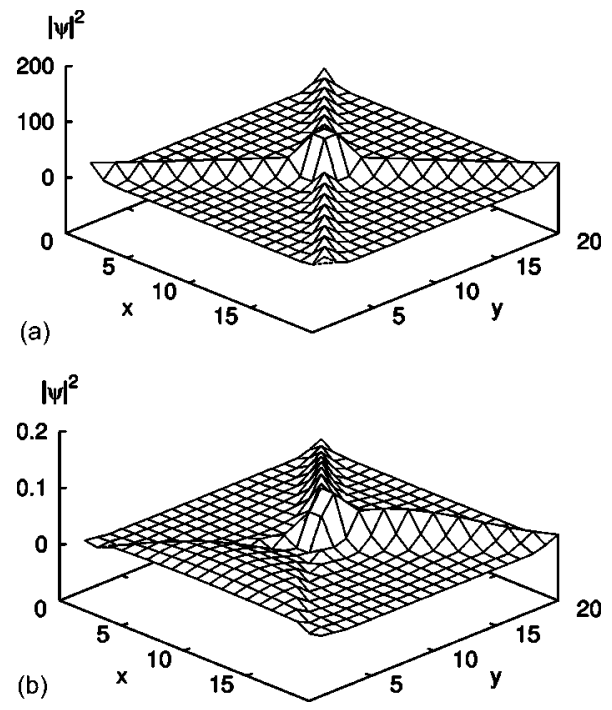

FIG. 4. Sample states of a particle traversing a $20 \times 20$ lattice with busbar connections. (a) Highly transmitting state with $E$ $=0.017$. (b) Highly reflecting state with $E=0.051$.

indeed it is close to a transmission minimum. For $L=6, \lambda$ $=5 / n(n=1,2,3,4)$, corresponding to $E \approx-1.62$ and 0.62 , also satisfy the condition and they are also close to a minima of $T$. In addition, at $\lambda=2(E=-2)$ we actually observe completely constructive interference for $L=2$ and 4 , where $L$ $-1=(1 / 2) \lambda$ and $L-1=(3 / 2) \lambda$, respectively, are satisfied. These observations suggest strong influences of interference on or near the connection boundaries on the overall transmission regarding the busbar connection though this boundary interference effect is far from providing a satisfactory explanation. In fact, since we have a discrete system with unit lattice constant rather than a continuous slit as in an optical system, it is not clear why $\lambda=1$ actually leads to destructive interference rather than the opposite (except for $L=2$ ). Of course, any influence of interference along the connection boundary must only be a part of the story since interference actually occurs throughout the bulk of the system (on most of which $\lambda$ is not even well defined) and since it must also compete with resonant transmission and reflection whenever the values of the incident particle's energies at the input chain fall near the eigenvalues of the isolated cluster.

Two sample states for a particle traversing a $20 \times 20$ lattice with busbar connections to the input and output chains are shown in Fig. 4. The busbars are connected at the $y=1$ and $y=20$ sides of the lattice. Shown in Fig. 4(a) is a highly transmitting state while Fig. 4(b) is a highly reflecting state. Notice that the difference in amplitudes between the states is several orders of magnitudes. In Fig. 4(b) strong destructive interference occurs in such a way that the state $\psi$ nearly vanishes within the lattice.

In conclusion, we find resonant transmission and reflection in the transport of a particle through finite square lattices whenever the particle's energy is near a doubly degenerate eigenvalue of the isolated lattice. The way the input and output chains are attached to the lattice influences the transport behavior of the incident particle. For point-to-point contacts particle transport is mostly transmitting but with trans- 
mission dips whenever resonance occurs. For busbar connections particle transport is mostly reflecting with transmission peaks whenever resonance also occurs. There are, however, peaks in transmission that cannot be accounted for by resonance. These peaks are the results of interference originating from the multiple connections in a busbar. The sharp fluctuations of the conductance in the $p=1$ limit, both as a function of the energy $E$ of the incident particle and the type of coupling chosen between the lattice and chains, will definitely affect the nature of transport whenever disorder is introduced, especially in the weak-disorder limit. However, whether these fluctuations have a significant effect on the existence or nature of the disputed localized-to-delocalized transition or whether they only affect transport at the weak disorder limit still remains to be explored. We are currently conducting such a study.

We would like to thank Y. Goldschmidt, Y. Lyanda-Geller, G. Baskaran, A. Finkelstein, L. Rokhinson, G. Giuliani, and N. Giordano for fruitful discussions.
[1] S. Kirkpatrick and T. P. Eggarter, Phys. Rev. B 6, 3598 (1972).

[2] P. de Gennes, P. Lafore, and J. Millot, J. Phys. Chem. Solids 11, 105 (1959).

[3] D. Stauffer and A. Aharony, Introduction to Percolation Theory, 2nd revised ed. (Taylor \& Francis, Bristol, PA 1994).

[4] A. Mookerjee, I. Dasgupta, and T. Saha, Int. J. Mod. Phys. B 9, 2989 (1995).

[5] D. Daboul, I. Chang, and A. Aharony, Eur. Phys. J. B 16, 303 (2000).
[6] G. Hałdaś, A. Kolek, and A. Stadler, Phys. Status Solidi B 230, 249 (2002).

[7] M. Büttiker, Y. Imry, R. Landauer, and S. Pinhas, Phys. Rev. B 31, 6207 (1985).

[8] W. Press, S. Teukolsky, W. Vetterling, and B. Flannery, Numerical Recipes in Fortran, 2nd ed. (Cambridge University Press, Cambridge, U.K., 1992).

[9] S. Datta, Electronic Transport in Mesoscopic Systems (Cambridge University Press, Cambridge, U.K., 1995). 